\title{
Zip and velcro bifurcations in competition models in ecology and economics
}

\author{
Jason A.C. Gallas ${ }^{1,2,3, a}$ \\ ${ }^{1}$ Max-Planck-Institut für Physik komplexer Systeme, 01187 Dresden, Germany \\ ${ }^{2}$ Complexity Sciences Center, 9225 Collins Avenue Suite 1208, Surfside, FL 33154, USA \\ ${ }^{3}$ Instituto de Altos Estudos, Rua Silvino Lopes 419-2502, 58039-190 João Pessoa, Brazil
}

Received 23 August 2019

Published online 26 March 2020

\begin{abstract}
During the last six years or so, a number of interesting papers discussed systems with line segments of equilibria, planes of equilibria, and with more general equilibrium configurations. This note draws attention to the fact that such equilibria were considered previously by Miklós Farkas (1932-2007), in papers published in 1984-2005. He called zip bifurcations those involving line segments of equilibria, and velcro bifurcations those involving planes of equilibria. We briefly describe prototypical situations involving zip and velcro bifurcations.
\end{abstract}

\section{Introduction}

About ten years ago it was realized that, in addition to the familiar chaotic attractors associated with saddle points, dynamical systems may also contain attractors not connected to such points, the so-called hidden attractors [1,2]. This startling finding induced considerable interest and a large number of publications concerning the properties of systems displaying no equilibria, chaotic systems with lines of equilibria, as well as systems containing planes and more general surfaces of equilibria.

For instance, by performing a systematic computer search among certain of families polynomial dynamical systems, Jafari and Sprott [3] found nine chaotic flows with quadratic nonlinearities which have the unusual feature of displaying a line segment of equilibrium points. Other similar polynomial flows were also investigated $[4,5]$. As remarked by these authors, such systems belong to a newly introduced category of chaotic systems with hidden attractors that are important and potentially problematic in real-life applications. Wang and Chen [6] reported on how to construct systems having any number of equilibria. Uyaroğlu and Kocamaz [7] investigated the control of a chaotic system having a line of equilibria using a passive control method. A chaotic flow with a plane of equilibria was investigated by Jafari et al. [8]. Very recently, Wu et al. [9] studied a neural memristor with infinite or without equilibrium, while Pham and coworkers presented a gallery of chaotic systems with an infinite number of equilibrium points [10] and reported simulations and an experimental implementation of a system with a line of equilibria but no linear term [11].

${ }^{a}$ e-mail: jgallas@pks.mpg.de 
The purpose of this note is to draw attention to the fact that systems with lines and surfaces of equilibria were considered previously by Miklós Farkas (19322007), in a series of papers published during the years 1984-2005. Farkas called zip bifurcations those involving line segments of equilibria, and velcro bifurcations those in systems with planes of equilibria. In the remainder of this note, we briefly review these concepts.

\section{Zip bifurcations}

Farkas introduced the concept of zip bifurcations in 1984 [12,13]. An extended and detailed presentation is given in his 1994 book Periodic Motions [14]. Farkas found zip bifurcations while studying the competition dynamics involving one prey and two predator species:

$$
\begin{aligned}
\dot{S} & =\gamma S\left(1-\frac{S}{K}\right)-\frac{m_{1} x_{1} S}{a_{1}+S}-\frac{m_{2} x_{2} S}{a_{2}+S}, \\
\dot{x}_{1} & =\frac{m_{1} x_{1} S}{a_{1}+S}-d_{1} x_{1}, \\
\dot{x}_{2} & =\frac{m_{2} x_{2} S}{a_{2}+S}-d_{2} x_{2} .
\end{aligned}
$$

In these equations, $x_{1}, x_{2}, S$ denote the population size of the two predator and the prey species, respectively. Clearly, in the absence of predators, the prey follow a standard logistic growth whose increase is controlled by $\gamma>0$. The carrying capacity of the environment with respect to the prey is $K>0$. The impact of the predators is assumed to be regulated by the Michaelis-Menten kinetics [15], where $m_{i}>0$, $d_{i}>0$, and $a_{i}$ are, respectively, the maximum birth rate, the death rate and the half saturation constant of the $i$ th predator.

Now, introduce auxiliary variables

$$
\lambda_{i}=\frac{a_{i} d_{i}}{m_{i}-d_{i}}, \quad \beta_{i}=m_{i}-d_{i}, \quad b_{i}=m_{i} / d_{i},
$$

and assume that $0<\lambda=\lambda_{1}=\lambda_{2}<K$, implying $\beta_{i}>0, b_{i}>1$. These definitions change equations (1)-(3) into

$$
\begin{aligned}
\dot{S} & =\gamma S\left(1-\frac{S}{K}\right)-\frac{m_{1} x_{1} S}{a_{1}+S}-\frac{m_{2} x_{2} S}{a_{2}+S}, \\
\dot{x}_{1} & =\beta_{1} x_{1} \frac{S-\lambda}{a_{1}+S}, \\
\dot{x}_{2} & =\beta_{2} x_{2} \frac{S-\lambda}{a_{2}+S} .
\end{aligned}
$$

Clearly, in the non-negative octant $\mathbb{R}_{+}^{3}$ the equilibria of the equations above are $(0,0,0),(K, 0,0)$ as well as the points on the straight line segment $L[14,16]$ :

$$
L=\left\{\left(\lambda, \xi_{1}, \xi_{2}\right) \in \mathbb{R}_{+}^{3} \mid \frac{m_{1} \xi_{1}}{a_{1}+\lambda}+\frac{m_{2} \xi_{2}}{a_{2}+\lambda}=\gamma\left(1-\frac{\lambda}{K}\right)\right\}
$$

To see what Farkas calls zip bifurcation, fix parameters as follows [16]:

$$
\gamma=\lambda=a_{2}=1, \quad a_{1}=m_{2}=2, \quad m_{1}=3,
$$


and consider the triplet of points

$$
P_{K}=\left(1,0,1-\frac{1}{K}\right), \quad M_{K}=\left(1,3-\frac{9}{K}, \frac{8}{K}-2\right), \quad Q_{K}=\left(1,1-\frac{1}{K}, 0\right),
$$

where $K \in(3,4)$. With the choices above, it is not difficult to see that the straight line segment $L$ connects the points $P_{K}$ and $Q_{K}$, and that the point $M_{k}$ is contained in the line $L$. As discussed in Section 7.4 of Farkas' book [14], the equilibria on $L$ located between $P_{K}$ and $M_{k}$ are unstable, while the equilibria between $M_{k}$ and $Q_{K}$ are stable. As $K$ increases from 3 to 4 , the point $M_{k}$ moves on the line from $P_{K}$ to $Q_{K}$, so that all points located on the left of $M_{k}$ become unstable. As $K$ is varied, the line $L$ undergoes a parallel displacement which, however, has no effect on the aforementioned scenario. For additional references and examples in more general contexts and the corresponding analysis, see Section 7.4 of Farkas' book [14].

More recent work by Ferreira and Rao deals with zip-bifurcation in a predatorprey model with diffusion [17], and in systems involving discrete delay $[18,19]$ and cross-diffusion [20]. Zip bifurcations are also discussed by Escobar-Callejas et al. [21] and Echeverri et al. [22].

\section{Velcro bifurcations}

Velcro bifurcations were considered in 2003 by Bocsó and Farkas [23], in the context of a political and economic rationality economic problem modelled by a set of four differential equations taking into account information concerning the problem spread among the people who support the political alternatives. In such model, velcro bifurcation occurs for specific parameter combinations destabilizing the equilibrium points when information spreads [23].

The model consists of the following equations:

$$
\begin{aligned}
\dot{v} & =\gamma v\left(1-\frac{y}{K}\right)-\sum_{i=1}^{3} m_{i} \frac{v}{a_{i}+v} u_{i}-M \frac{v}{A+v} \frac{u_{1}}{u_{2}}, \\
\dot{u}_{1} & =m_{1} \frac{v}{v+a_{1}} u_{1}-d_{1} u_{1}, \\
\dot{u}_{2} & =m_{2} \frac{v}{v+a_{2}} u_{1}-d_{2} u_{2}, \\
\dot{u}_{3} & =m_{3} \frac{v}{v+a_{3}} u_{1}-d_{3} u_{3} .
\end{aligned}
$$

The model has similarities with the previous one, control parameters obey similar relations but have rather different meanings [23] which are of no concern for our purpose here.

The last three equations of the model above may be simplified to

$$
\dot{u}_{i}=\beta_{i} \frac{v-\lambda_{i}}{v+a_{i}} u_{i}, \quad i=1,2,3
$$

where, similarly as before,

$$
\beta_{i}=m_{i}-d_{i}, \quad b_{i}=m_{i} / d_{i}, \quad \lambda_{i}=\frac{a_{i} d_{i}}{\beta_{i}}=\frac{a_{i}}{b_{i}-1} .
$$


Under specific but realistic relations of the parameters the equilibrium points of the system form a surface [23]

$$
\begin{aligned}
S=\left\{\left(v, u_{1}, u_{2}, u_{3}\right) \in \mathbb{R}^{4} \quad \mid \quad\right. & =\lambda, u_{1}, u_{2}, u_{3}>0 \\
& \left.\gamma\left(1-\frac{v}{K}\right)=\sum_{i=1}^{3}\left(\frac{m_{i} u_{i}}{a_{i}+v}+\frac{M}{A+v} \frac{u_{1}}{u_{2}}\right)\right\}
\end{aligned}
$$

In the above context, the dynamics of the velcro bifurcations is defined as a sort of generalized zip bifurcations, as spelled out in Theorem 2 of Bocsó and Farkas [23]:

"The surface $S$ is divided into two parts by a curve $g$; the equilibria on the upper part of $S$ are still stable, and this part is an attractor of the system (in the sense described in [14]); the equilibria on the lower parts are already unstable. The curve $g$ moves upwards as $K$ is increased leaving behind the destabilized equilibria."

Velcro bifurcations were also reported in 2005 by Farkas, Sáez, and Szántó in competition models with generalized Holling functional response [24,25]. Specifically, their basic model is given by the equations:

$$
\begin{aligned}
& \dot{S}=r S\left(1-\frac{S}{K}\right)-\sum_{i=1}^{3} m_{i} x_{i} \frac{S^{n}}{a_{i}^{n}+S^{n}}, \\
& \dot{x}_{i}=m_{i} x_{i} \frac{S^{n}}{a_{i}^{n}+S^{n}}-d_{i} x_{i}, \quad i=1,2,3
\end{aligned}
$$

where $n>2$ is an integer. For a detailed analysis and several figures of the equilibrium surfaces, consult the original article [24,25].

\section{Conclusions and outlook}

The purpose of this note is to bring the works of Farkas and co-workers to the attention of researchers working in the interesting field of systems with line and surfaces of equilibria. As remarked in 1996 by Freedman [26], "For those who don't know, zip bifurcations were first discovered by Professor Farkas, describing how a singular curve unfolds into periodic solutions when a parameter changes, just like a zipper opening up."

It is interesting to note that most of the recent systems found to contain lines and surfaces of equilibria which deal with interesting but abstract polynomial systems, arising from exhaustive computer searches, that are not yet associated with any applications. In contrast, Farkas and co-workers found zip and velcro bifurcations in standard systems that contain typical nonlinearities of the sort encountered in popular models used in biology and economy. A publication list containing 76 works of Farkas is given in reference [16], while a list with 78 works, signed by "students and colleagues", is given in reference [27]. A special issue of the journal Differential Equations and Dynamics Systems was dedicated to Farkas [28].

As it is clear from the literature, there is presently great interest in investigating changes in the topology of attractors not restricted to small neighborhoods of points. The works of Farkas still contain a plethora of theorems and unexplored materials that deserve attention, and that will certainly contribute to the understanding of the rich dynamics of systems with equilibria defined by extended mathematical structures. 
Open access funding provided by Projekt DEAL. Work done in the framework of an Advanced Study Group on Forecasting with Lyapunov vectors, at the Max-Planck Institute for the Physics of Complex Systems, Dresden. The author was supported by CNPq, Brazil. The article processing charges for this open-access publication were covered by the Max Planck Society.

Open Access This is an open access article distributed under the terms of the Creative Commons Attribution License (https://creativecommons.org/licenses/by/4.0), which permits unrestricted use, distribution, and reproduction in any medium, provided the original work is properly cited.

\section{References}

1. G.A. Leonov, N.N. Kuznetsov, V.I. Vagaitsev, Phys. Lett. A 375, 2230 (2011)

2. D. Dudowski, S. Jafari, T. Kapitaniak, N.V. Kuznetsov, G.A. Leonov, A. Prasad, Phys. Rep. 637, 1 (2016)

3. S. Jafari, J.C. Sprott, Chaos, Solitons Fractals 57, 79 (2013)

4. C. Li and J.C. Sprott, Phys. Lett. A 378, 178 (2014)

5. C. Li, J.C. Sprott, W. Thio, J. Exp. Theory Phys. 118, 494 (2014)

6. X. Wang, G. Chen, Nonlinear Dyn. 71, 429 (2013)

7. Y. Uyaroğlu, U.E. Kocamaz, Chaot. Model. Simul. 4, 277 (2015)

8. S. Jafari, J.C. Sprott, M. Molaie, Int. J. Bifurc. Chaos 26, 1650098 (2016)

9. F. Wu, G. Zhang, J. Ma, Eur. Phys. J. Special Topics 228, 1527 (2019)

10. V.T. Pham, S. Jafari, C. Volos, T. Kapitaniak, Chaos, Solitons Fractals 93, 58 (2016)

11. V.T. Pham, S. Jafari, C. Volos, L. Fortuna, Chaos, Solitons Fractals 120, 213 (2019)

12. M. Farkas, Nonlinear Anal. TMA 8, 1295 (1984)

13. M. Farkas, Competitive exclusion by zip bifurcations, in Lecture Notes on Economics and Mathematical Systems, edited by A.B. Kurzhanski, K. Sigmund (Springer, Berlin, 1987), Vol. 287, pp. 165-178

14. M. Farkas, Periodic motions (Springer, New York, 1994)

15. K.A. Johnson, R.S. Goody, Biochemistry 50, 8264 (2011)

16. Anonymous, Miklós Farkas obituary, Period. Math. Hung. 56, 1 (2008)

17. J.D. Ferreira, L.A.F. de Oliveira, Differ. Equ. Dyn. Syst. 17, 37 (2009)

18. M. Farkas, J.D. Ferreira, P.C.C. Tabares, Annales Univ. Sci. Budapest, Sect. Comp. 25, $53(2005)$

19. J.D. Ferreira, V.S.H. Rao, Proc. R. Soc. Edinburgh 143A, 1209 (2013)

20. J.D. Ferreira, S.H. da Silva, V.S.H. Rao, Physica D 400, 132141 (2019)

21. C.M. Escobar-Callejas, J.R. Gonzáles-Granada, A.E. Posso-Agudelo, Ingenieria y Ciencia 6, 11 (2010) (in Spanish)

22. L.F. Echeverri, O.I. Giraldo, E. Zarrazola, Revista Integración, Univ. Industrial de Santander 35, 127 (2017)

23. A. Bocsó, M. Farkas, Appl. Math. Comput. 140, 381 (2003)

24. M. Farkas, E. Sáez, I. Szántó, Miskolc Math. Notes 6, 185 (2005)

25. E. Sáez, E. Stange, I. Szántó, SIAM J. Appl. Dyn. Syst. 5, 1 (2006)

26. H.I. Freedman, Bull. Am. Math. Soc. 33, 119 (1996)

27. Anonymous, Alkalmazott Matematikai Lapok 25, 155 (2008) (in Hungarian)

28. J.D. Ferreira, G. Stépán, Differ. Equ. Dyn. Syst. 17, 1 (2009) 